\title{
RESEARCH ON HIGH ACCURACY DETECTION OF RED TIDE HYPERSPECRRAL BASED ON DEEP LEARNING CNN
}

\author{
Yabin $\mathrm{HU}{ }^{1,2}$, Yi $\mathrm{MA}^{2 *}$, Jubai $\mathrm{AN}^{1}$ \\ ${ }^{1}$ Information Science and Technology College, Dalian Maritime University, Dalian 116026, China, hu_yabin@163.com, \\ jubaian@dlmu.cn; \\ ${ }^{2}$ The First Institute of Oceanography, SOA, Qingdao 266061, China, mayimail@fio.org.cn
}

\section{Commission III, WG III/4}

KEY WORDS : Red Tide, CNN, Hyperspectral, Remote Sensing, Glint

\begin{abstract}
:
Increasing frequency in red tide outbreaks has been reported around the world. It is of great concern due to not only their adverse effects on human health and marine organisms, but also their impacts on the economy of the affected areas. this paper put forward a high accuracy detection method based on a fully-connected deep CNN detection model with 8-layers to monitor red tide in hyperspectral remote sensing images, then make a discussion of the glint suppression method for improving the accuracy of red tide detection. The results show that the proposed CNN hyperspectral detection model can detect red tide accurately and effectively. The red tide detection accuracy of the proposed CNN model based on original image and filter-image is $95.58 \%$ and $97.45 \%$, respectively, and compared with the SVM method, the CNN detection accuracy is increased by $7.52 \%$ and $2.25 \%$. Compared with SVM method base on original image, the red tide CNN detection accuracy based on filter-image increased by $8.62 \%$ and $6.37 \%$. It also indicates that the image glint affects the accuracy of red tide detection seriously.
\end{abstract}

\section{INTRODUCE}

Red tide is a kind of oceanic disasters caused by the explosive propagation or aggregation of plankton in sea water under certain conditions, which can cause the discoloration of the seawater. Seawater presents different colors due to different types of red tides during the red tide outbreak, but some red tide creatures do not cause a color anomaly phenomenon in the seawater. Therefore, it is difficult to accurately conclude that there is an occurrence of red tide based on the color of seawater change only. Red tide has become one of the global marine disasters, it not only affects the ecological environment of water, but also brings significant economic losses of fishery resources and production. Additionally, red tide outbreaks could force the shutdown of desalination plants, which pose a major threat to the potable water supply (Berktay, A, 2011a). In recent years, the degree of eutrophication of certain coastal water in China has become serious, which lead to occurrence of red tide with large scale frequently. Red tide causes huge losses of the coastal economy and endangers the human living environment. Therefore, the rapid and accurate detection of red tide is basic for the prevention and control of red tide disasters. Due to the species in the sea is diverse, the color of red tide water is different. It is different to determine whether the red tide is emerging according to the color change of seawater.

Red tide, also known as harmful algal bloom, is caused by proliferation of a toxic or nuisance algae species and has been a preeminent topic of world-wide research communities for several decades (Cullen et al., 1997, Kahru et al., 2000, Ahn et al., 2006, Gower et al., 2008, Wang et al., 2011a, Hu and Feng, 2017). Red tide monitoring is realized in two ways: in-situ measurement and

*Corresponding author : Yi MA, E-mail: mayimail@fio.org.cn 
remote sensing monitoring (MA et al., 2002, Xu et al., 2014, Zhao et al., 2014b). it is very essential to detect and forecast their movement using currently available remote sensing technology because traditional ship-based field sampling and analysis are very limited in both space and temporal frequency. Remote sensing has the advantages of wide spatial coverage and short repetition period, which can monitor large area of red tide effectively (MA et al., 2002, Ahn et al., 2006). Remote sensing includes satellite remote sensing and aerial remote sensing, of which the former has shortcomings including longer observation period, lower spatial resolution, and fewer wave bands of the sensor than those of the latter. Hyperspectral remote sensing technology is the frontier of aerial remote sensing development. The spectral range of hyperspectral images is covering visible and near infrared band, which spectral resolution reaches up to nanometer level, so it is suitable to identify red ride (MA et al., 2006, ZHAO et al., 2008). The deep learning methods presents high robustness and efficiency in image processing (Hinton et al. 2014). Convolution Neural Network (CNN) (Hinton et al. 2006), a deep learning method, has the characteristics of image displacement and rotation invariance, which has been applied in image processing successfully (Cao et al. 2016). However, it is weak in the red detection. Simultaneously, it is noted that sea surface glint in hyperspectral images interferes the spectral information of the target seawater, which will affect the effective detection of red tide. The influence of sea surface glint on red tide detection is also the research direction.

Therefore, this paper put forward a high accuracy detection method based on deep learning algorithm to monitor red tide in hyperspectral remote sensing images, then make a discussion of the glint suppression method for improving the accuracy of red tide detection.

\section{DATAS AND METHODS}

\subsection{Hyperspectral Image}

The red tide hyperspectral image used in this paper is acquired from China Marine Surveillance aircraft carrying PHI (PushBroom Hyperspectral Imager) on August 12, 2001. The PHI hyperspectral image covers the Bayuquan area of Liaodong Bay in Bohai Sea. There are 124 bands in the PHI image cubic with wavelength ranging from $383.3 \mathrm{~nm}$ to $881.4 \mathrm{~nm}$, the size is $652 * 1000$, and the spectral resolution is better than $5 \mathrm{~nm}$, which has the ability to distinguish red tide from normal seawater. We extracted the red tide interpretation map by using humancomputer interaction method to validate detection accuracy. PHI hyperspectral image and red tide verification map are shown in Figure 1.
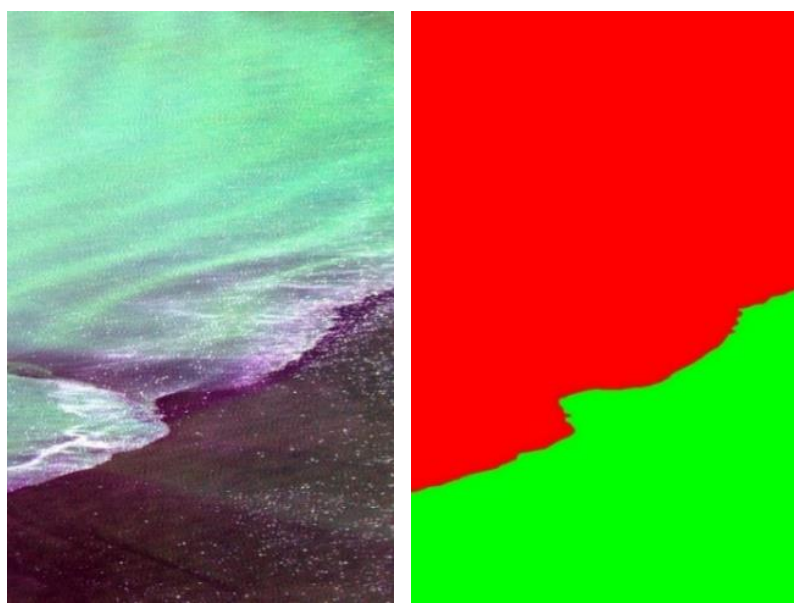

Figure 1 the map of PHI spectral remote sensing image (R:10, G:60, B:100) and the red tide validation map (red: red tide, green: seawater)

\subsection{Red Ride CNN Detection Model}

This paper developed a fully-connected deep CNN detection model with 8-layers to detect red tide based on PHI hyperspectral image. The model includes 1 input layer, 2 convolution layers, 2 drop samples layers, 2 fully-connected layers and 1 output layer (Figure 2)

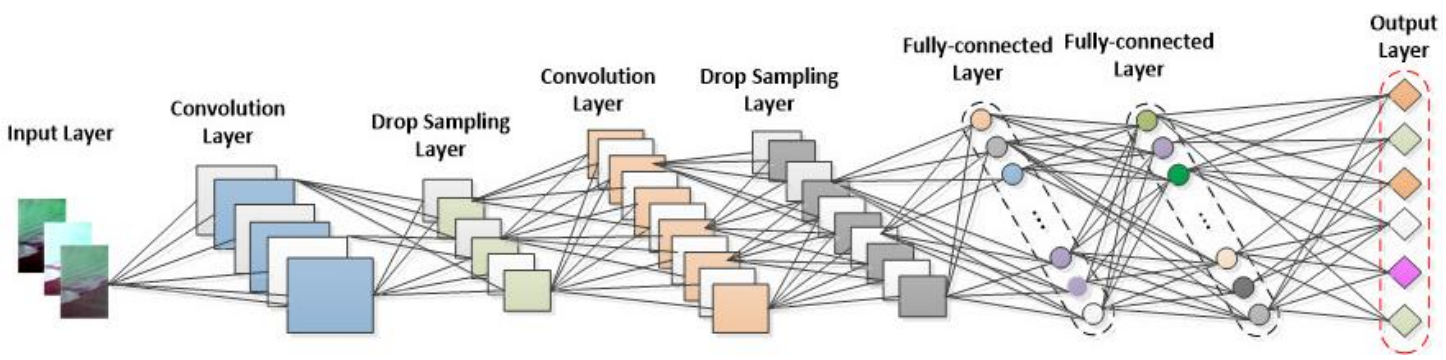

Figure 2 The structure of $\mathrm{CNN}$ red tide detection model 
We take the spectral feature information as the input information in $\mathrm{CNN}$ model. The spectral feature information contains the original spectra and the first derivative spectra. The first derivative spectra of the original spectral reflectance, that is, the slope value of the spectral curve, can reflect the rate of change of the original spectral curve, where the positive value represents the band where the reflectance increases the most, and the negative value represents the band where the reflectivity deceleration is the largest. The first derivative spectra can be expressed by the formula (1)

$\frac{d L}{d \lambda}=T \cdot E \cdot \frac{d \rho}{d \lambda}+\rho \cdot T \cdot \frac{d E}{d \lambda}+E \cdot \rho \cdot \frac{d T}{d \lambda}+\frac{d L p}{d \lambda}$

Where $L$ is the energy of imaging spectral image

$\rho$ is the ground object reflectivity

$T$ is the atmospheric transmittance.

$E$ represents the solar irradiance

$L p$ represents the process radiation

$\lambda$ is the wavelength

The paper takes the balance of the samples into account, we randomly sample a certain number of pixels from the hyperspectral image for training and use the rest to evaluate the performance of the proposed network. we select 6750 training samples and 2652 testing samples of red tide and seawater for the

CNN detection model. For each training pixel, we crop surrounding $3 \times 3$ neighboring pixels for learning convolutional filters, and the convolutional layer is activated by the sigmiod function. The down-sampling layer adopts the maximum pooling method, and the size is $2 \times 2$. The fully-connected layer is used for high level reasoning. Neurons in this layer have full connections to all activations in the batch normalization layer so as to integrate information across all locations in all the feature maps of the previous layer. The dropput used to avoid overfitting. The output layers are activated by the softmax function. The number of units in the output layer is set as the number of classes. When a pixel is fed to the network, it is classified to the neuron with maximum outputs.

\section{RESULTS AND ANALYSIS}

In order to validate the influence of red tide detection from the glint image, we adapt the median filter algorithm, the filter size is $3 \times 3$, then carry out detecting red tide based on the denoise image. Then we used the red tide interpretation map to validate the accuracy of red tide detection. The effectiveness of the proposed method is evaluated by comparing it with the state-of-the-art classification approach SVM. The detection results are shown in Figure 3 and Table 1.
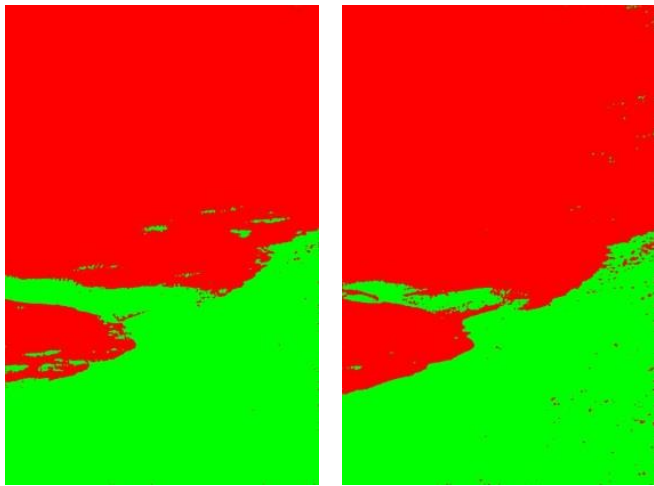

Figure 3 the red tide detection results (From left to right: SVM, CNN, Filter-SVM, Filter-CNN)

\begin{tabular}{ccccc}
\hline Types & SVM & CNN & Filter-SVM & Filter-CNN \\
\hline Red tide & 83.41 & 95.71 & 92.91 & 96.80 \\
Seawater & 99.27 & 95.35 & 87.84 & 98.81 \\
OA & 88.83 & 95.58 & 95.20 & 97.45 \\
Kappa & 0.76 & 0.90 & 0.89 & 0.94
\end{tabular}

Table 1 The red tide detection accuracy $(\%)$ 
The results show that the red tide detection accuracy of the proposed CNN model and SVM method is $95.58 \%$ and $88.83 \%$, and the Kappa coefficient is 0.90 and 0.76 , respectively, the CNN detection accuracy is increased by $7.52 \%$. Base on the PHI hyperspectral image after median filter, the red tide detection accuracy of the proposed CNN model and SVM is $97.45 \%$ and $95.20 \%$, respectively, and the Kappa coefficient is 0.94 and 0.89 , the $\mathrm{CNN}$ detection accuracy is increased by $2.25 \%$. Compared with SVM method base on original image, the red tide detection accuracy increased by $8.62 \%$ and $6.37 \%$. The accuracy of CNN red tide detection based on original image is similar with that of SVM red tide detection based on filter-image, the reason is that the convolution process in $\mathrm{CNN}$ detection model has a filtering effect, so the CNN model can suppress sea surface glint in a certain extent. Compared with the red tide detection accuracy of SVM method, the accuracy of CNN model is improved by $2.25 \%$ base on filter-image. The red tide detection method based deep learning CNN shows that it is a practical approach for red tide detection in challenging conditions of sea surface glint in image. It indicates that the proposed CNN hyperspectral detection model can detect red tide accurately and effectively, and the image glint affects the accuracy of red tide detection seriously.

\section{CONCLUSION AND DISCUSSION}

Red tide is caused by proliferation of a toxic or nuisance algae species and has been a preeminent topic of world-wide research communities. This paper developed a red tide CNN detection model with fully-connected 8-layers based on PHI hyperspectral images, which combined original spectral feature and the first derivative spectral feature information. We compared it with the SVM classification algorithm. At the same time, we evaluated the accuracy of the hyperspectral red tide detection by the remote sensing interpretation map. The results show that the proposed CNN hyperspectral detection model can detect red tide accurately and effectively. The red tide detection accuracy of the proposed $\mathrm{CNN}$ model based on original image and filter-image is $95.58 \%$ and $97.45 \%$, respectively, and compared with the SVM method, the $\mathrm{CNN}$ detection accuracy is increased by $7.52 \%$ and $2.25 \%$. Compared with SVM method base on original image, the red tide CNN detection accuracy based on filter-image increased by $8.62 \%$ and $6.37 \%$. We will explore the impact of red tide hyperspectral detection by different glint suppression methods in the future work.

\section{ACKNOWLEDGMENTS}

This investigative study is fully funded by National Natural Science Foundation of China (No. 41206172), the National Natural Science Foundation of China (No. 41506204) and the National Natural Science Foundation of China (No. 61601133). Acknowledgements of support for the paper are welcome.

\section{REFERENCES}

Ahn, Y.-H., Shanmugam, P., 2006. Detecting the red tide algal blooms from satellite ocean color observations in optically complex Northeast-Asia coastal waters. Remote Sensing of Environment, 103, pp. 419-437.

Berktay, A., 2011a. Environmental approach and influence of red tide to desalination process in the Middle East region. International Journal of Chemical and Environmental

Engineering, 2 (3), pp. 183-188.

Cao Linlin, Li Haitao, Han Yanshun, et al., 2016. Application of convolutional neural networks in nlassification of high resolution remote sensing imagery. Science of Surveying and Mapping, 41(9): pp. 170-175.

Chuanmin Hu, Lian Feng, 2017. Modified MODIS fluorescence line height data product to improve image interpretation for red tide monitoring in the eastern Gulf of Mexico. Journal of Applied Remote Sensing,11(1), pp. 1-14.

Cullen, J.J., Ciotti, A.M., Davis, R.R., Lewis, M.R., 1997. Optical detection and assessment of algal blooms. Limnology and Oceanography, 42 (5), pp. 1223-1239.

\section{G. E. Hinton and R. R. Salakhutdinov, 2006. Reducing the} dimensionality of data with neural networks. Science, 313(5786): pp. 504-507.

Gower, J.F.R., King, S.A., Goncalves, P., 2008. Global monitoring of plankton blooms using MERIS MCI. International Journal of Remote Sensing, 29, pp. 6209-6216. 
Hinton G E, Osindero S, Teh Y W, 2014. A fast learning algorithm for deep belief nets. Neural Computation, 18(7), pp. 1527-1554.

Jun Zhao, Hosni Ghedira, 2014. Monitoring red tide with satellite imagery and numerical models: a case study in the Arabian Gulf. Marine Pollution Bulletin,79(1-2), pp. 305-313.

Kahru, M., Leppänen, J.M., Rud, O., Savchuk, O.P., 2000. Cyanobacteria blooms in the Gulf of Finland triggered by saltwater inflow into the Baltic Sea. Marine Ecology Progress Series, 207, pp. 13-18.

MA Yi, ZHANG Jie, CUI Tingwei, 2006. Research on airborne hyperspectral identification of red tide organism dominant species based on SVM. Spectroscopy and Spectral Analysis,12(26), pp. 2302-2305.

MA Yi, ZHANG Jie, ZHANG Hande, LIU Yuzhong, 2002. Advances in application research on the marine airborne hyperspectral remote sensing in China. Advances in Marine Science, 20(4): pp. 94-98.

Wang, G., Zhou, W., Cao, W., Yin, J., Yang, Y., Sun, Z., Zhang, Y., Zhao, J., 2011a. Variations of particulate organic carbon and its relationship with bio-optical properties during a phytoplankton bloom in the Pearl River estuary. Marine Pollution Bulletin, 62 (9), pp. 1939-1947.

Xu Xiaohui, Pan Delu, Mao Zhihua, Tao Bangyi, 2014b. A new algorithm based on the background field for red tide monitoring in the East China Sea. The Chinese Society of Oceanography and Springer-Verlag Berlin Heidelberg 2014, 33(5): pp. 62-71.

ZHAO Wencang, WEI Hongli, SHI Chagnjiang, JI Guangrong, G.,2008. The recognition of ocean red tide with hyper-spectralimage based on EMD. Chinese Journal of Oceanology and Limnology, 26(2), pp. 137-141. 